Published in final edited form as:

Nat Clin Pract Gastroenterol Hepatol. 2005 August ; 2(8): 352-353. doi:10.1038/ncpgasthep0241.

\title{
Should African Americans be screened for colorectal cancer at an earlier age?
}

\author{
J M Carethers [Professor] \\ Medicine at the University of California, San Diego, and a member of the Veterans Administration \\ Research Service, San Diego, CA, USA
}

\section{Keywords}

age; African Americans; colonoscopy; colorectal cancer; screening

Original article Agrawal et al. (2005) Colorectal cancer in African Americans. Am J Gastroenterol 100: 515-523

\section{SYNOPSIS}

\section{BACKGROUND}

Incidence, mortality and morbidity rates of colorectal cancer are higher in AFRICAN AMERICANS compared with the general population but the reasons for this trend are unclear.

\section{OBJECTIVES}

To review the literature on colorectal cancer screening with particular regard to African Americans. To create recommendations for the assessment, screening and diagnosis of colorectal cancer in this group.

\section{DESIGN AND INTERVENTION}

Studies on colorectal cancer screening in the general population and in African Americans were identified and analyzed. Data on the incidence of cancer, survival rates, diagnostic methods, site of cancer distribution and barriers to screening within populations were analyzed. Recommendations were created based on the evidence obtained.

\section{OUTCOME MEASURES}

The main outcome was whether the screening and management of colorectal cancer in African Americans should be performed differently to that of the general population.

\footnotetext{
Correspondence. GI Section, 111D, VA San Diego Healthcare, System, 3350 La Jolla Village Drive, San Diego, CA 92161, USA, jcarethers@ucsd.edu.

Competing interests

The author declared he had no competing interests.
} 


\section{RESULTS}

The incidence of colorectal cancer was 12.3\% higher for African Americans compared with Whites between 1996 and 2000. A higher proportion of African Americans present with colorectal cancer under 50 years of age compared with Whites (10.6\% versus 5.5\%). Fiveyear survival rate is lower in African Americans compared with Whites (56\% versus 63\%). Distribution of colorectal cancer is more likely to be right-sided and proximal in African Americans. Colonoscopy is significantly more effective than other screening methods for the detection of small cancers. African Americans are $18 \%$ less likely to receive colonoscopy, and 39\% less likely to receive flexible sigmoidoscopy compared with Whites. African American patients had lower rates of colorectal cancer screening with fecal occult blood test compared with Whites. Fear of finding cancer, coupled with the socioeconomic and social consequences of cancer, are barriers to screening for non-Whites. Procedural related pain, feelings of violation, embarrassment or shame, inadequate patient information and physician mistrust are also factors. Income, education and health insurance affect screening participation rates (24\% of African Americans live in poverty compared with $8 \%$ of Whites).

\section{CONCLUSION}

Colorectal cancer develops at a younger age in African Americans compared with Whites. Colorectal cancer screening should begin at 45 years old in African Americans.

Colonoscopy is the preferred modality for colorectal cancer screening, particularly in African American patients, owing to the site distribution of colorectal cancer in this group. Awareness of the differences in colorectal cancer screening for African American patients should be raised amongst physicians and communities. Culturally sensitive educational programs regarding colorectal cancer screening for African Americans should be implemented.

\section{COMMENTARY}

\section{John M Carethers}

Colorectal cancer is the second leading cause of cancer death in the US. Data predict that 145,290 persons will be afflicted and 56,290 deaths will occur from the disease in 2005. ${ }^{1}$ Recommendations indicate that colorectal cancer screening for those at average risk should begin at 50 years of age. ${ }^{2}$ This recommendation is based on the dramatic increase in the cumulative incidence of colorectal cancers after this age, coupled with evidence from other screening modalities such as fecal occult blood tests, sigmoidoscopy or colonoscopy that can detect cancers at earlier stages or as precursor adenomatous polyps. Identifying and removing colonic neoplasms found as a result of these screening tests improves the prognosis of patients who otherwise might have progressed to advanced disease.

There are groups of patients for which these screening recommendations do not apply, owing to their higher risk for developing colorectal cancer. These include patients with FAMLIAL ADENOMATOUS POLYPosIS, LYNCH SYNDROME, and those with inflammatory bowel disease. These patients are at higher risk because the biology and epidemiology of their conditions allow 
their cancers to develop earlier in life, or at a more rapid pace than those without the disease. $^{2}$

African Americans also possess epidemiologic factors that put them at above average risk for colorectal cancer. African Americans have the highest incidence and death rates for colorectal cancer when compared to White, Asian, American Indian, and Hispanic or Latino groups. ${ }^{1,3}$ Colorectal tumors in African Americans are more likely to have distant spread (24\% versus 19\% for Whites), and are less likely to be localized (where cure rates are the highest). Consequently, the 5-year survival rates for African Americans with colorectal cancer are lower when compared with other groups. ${ }^{1}$ Furthermore, a higher proportion of African Americans under the age of 50 have colorectal cancer compared with Whites (10.6\% versus 5.5\%). ${ }^{3}$ Although differences in the biology of colorectal cancer between ethnic groups have not been adequately studied, Surveillance, Survey of Epidemiology and End Results (SEER), and other data indicate a more right-sided colonic distribution of colorectal cancers in African Americans relative to other groups. ${ }^{4}$

Agrawal et al. have made recommendations for colorectal cancer screening in African Americans: these include initiating colorectal cancer screening in this group at 45 years old instead of 50 years old; the use of colonoscopy over other screening modalities because of the prevalence of proximal colonic cancers; the development of educational programs for gastroenterologists, to make them aware of the issues of cancer behavior in African Americans; and the inclusion of culturally sensitive health educational programs for African American patients. These recommendations are sound, based on the published evidence. Gastroenterologists are generally aware of increased colorectal cancer risk in relation to familial adenomatous polyposis and inflammatory bowel disease, but the risks outlined for African Americans are less well known. In individuals at higher risk, screening for colorectal cancer should begin before the age of significant increases in cancer incidence for that group, and should be adjusted by the observed biology or epidemiology of the risk group. This approach has already been applied to certain ethnic groups for prostate and breast cancer screening programs. ${ }^{5}$

\section{Acknowledgments}

The synopsis was written by Rachel Jones, Associate Editor, Nature Clinical Practice.

\section{GLOSSARY}
AFRICAN
AMERICANS
FAMILIAL
ADENOMATOUS
POLYPOSIS

LYNCH
SYNDROME
Persons born in the US having ancestral origins in any of the black racial groups of Africa

A condition in which multiple adenomatous polyps develop in the colon: patients are at nearly $100 \%$ risk for colorectal cancer as a result of a germline mutation in the $A P C$ autosomal dominant) or $M Y H$ (autosomal recessive) genes

Autosomal dominant inheritance of mutated DNA mismatch repair genes $h M L H 1, h M S H 2, h M S H 6, h P M S 2$ ) resulting in 
high risk for colorectal cancer and cancers of the female reproductive, urinary and upper gastrointestinal tracts

\section{References}

1. Jemal A, et al. Cancer Statitiscs. CA Cancer J Clin. 2005; 55:10-30. [PubMed: 15661684]

2. Winawer $\mathrm{S}$, et al. Colorectal cancer screening and surveillance: clinical guidelines and rationaleupdate based on new evidence. Gastroenterology. 2003; 124:544-560. [PubMed: 12557158]

3. Theuer CP, et al. Racial and ethnic colorectal cancer patterns affect the cost-effectiveness of colorectal cancer screening in the United States. Gastroenterology. 2001; 120:848-856. [PubMed: 11231939]

4. Troisi RJ, et al. Incidence of colorectal carcinoma in the US. An update of trends by gender, race, age, subsite, and stage, 1975-1994. Cancer. 1999; 85:1670-1676. [PubMed: 10223559]

5. Smith RA, et al. American Cancer Society guidelines for the early detection of cancer. CA Cancer J Clin. 2005; 55:31-41. [PubMed: 15661685] 


\section{PRACTICE POINT}

Colorectal cancer screening by colonoscopy should begin at 45 years of age in African Americans 